\title{
26433 - RIGHT VENTRICULAR MYOCARDIAL PERFORMANCE IN VALVULAR SURGERY
}

\section{André Y. Denault MD FRCPC, François Haddad, MD; Pierre Couture, MD FRCPC; Michel Pellerin, Jean-Claude Tardif, MD FRCPC; Montreal Heart Institute, Montreal, QUEBEC, Canada}

INTRODUCTION: Right ventricular (RV) function plays an important role in the survival and in the postoperative course of patients with mitral and aortic valve disease. Right ventricular myocardial performance index (RV MPI), which is a measure of global RV function, could be a useful predictor of outcome in mitral and aortic valve surgery. Our objective was to study the relationship between preoperative RV MPI and difficult separation from bypass (DSB) following aortic or mitral valve surgery.

METHODS: We prospectively measured RV MPI, after induction of anesthesia, in 30 consecutive patients undergoing corrective mitral and aortic valve surgery. The RV MPI (normal value $=0.28 \pm 0.04$ ) was defined as the sum of isovolumic contraction time and isovolumic relaxation time divided by the ejection time. RV MPI was measured using both pulse wave and tissue Doppler imaging. Difficult separation from bypass represented the primary endpoint. DSB was predefined as a systolic arterial pressure $<80$ $\mathrm{mmHg}$ (confirmed with aortic or femoral pressure), and diastolic pulmonary artery pressure $>15 \mathrm{mmHg}$ during progressive separation from CPB resulting in reintroduction of extracorporeal circulation, insertion of an intra-aortic balloon pump or significant inotropic support of cardiac function (norepinephrine infusion equal or greater than 7 $\mathrm{mg} / \mathrm{kg} / \mathrm{min}$ or equal or greater than 2 inotropic agents with norepinephrine infusion equal or greater than $4 \mathrm{mg} / \mathrm{kg} / \mathrm{min}$ ).

RESULTS: In 30 consecutive patients, 16 women, 14 men, the mean age was $67 \pm 7$ years, NYHA was $2.7 \pm 0.6$, the Parsonnet score was $25 \pm 7$, 14 patients underwent aortic valve replacement, 9 patients underwent mitral valve replacement, 3 patients had mitral valve repair and 4 patients underwent combined valve replacement. The primary endpoint, DSB, occurred in 14 patients (47\%). Chi-square testing demonstrated that the primary endpoint was significantly associated with preoperative RV MPI $>0.40(\mathrm{p}<0.05)$. The sensitivity, specificity, positive predictive value and negative predictive value of a RV MPI $>0.40$ was $67 \%, 85 \%, 80 \%$ and $72 \%$ respectively. In our preliminary study, preoperative RV MPI was a better predictor of difficult weaning than age, Parsonnet score, pulmonary hypertension, cardiac index or bypass time.

CONCLUSIONS: Our preliminary study suggests that RV MPI, as an index of global RV function, could be a potential useful predictor of DSB in patients undergoing aortic or mitral valve surgery. 